\title{
La mobilité résidentielle comme cause et conséquence de vulnérabilité chez les personnes âgées à La Réunion
}

Residential mobility as a cause and consequence of vulnerability among the elderly in Reunion Island

\section{Armelle Klein et Frédéric Sandron}

\section{(2) OpenEdition} Journals

Édition électronique

URL : https://journals.openedition.org/popvuln/850

DOI : $10.4000 /$ popvuln. 850

ISSN : 2650-7684

Éditeur

LIR3S - Laboratoire Interdisciplinaire de Recherche "Sociétés Sensibilités Soin" (UMR 7366 CNRS-uB)

Référence électronique

Armelle Klein et Frédéric Sandron, «La mobilité résidentielle comme cause et conséquence de vulnérabilité chez les personnes âgées à La Réunion », Populations vulnérables [En ligne], 7 | 2021, mis en ligne le 01 décembre 2021, consulté le 26 février 2022. URL : http://journals.openedition.org/ popvuln/850 ; DOI : https://doi.org/10.4000/popvuln.850

Ce document a été généré automatiquement le 26 février 2022.

Les contenus de la revue Populations vulnérables sont mis à disposition selon les termes de la Licence Creative Commons Attribution 4.0 International 


\title{
La mobilité résidentielle comme cause et conséquence de vulnérabilité chez les personnes âgées à La Réunion
}

\author{
Residential mobility as a cause and consequence of vulnerability among the \\ elderly in Reunion Island
}

Armelle Klein et Frédéric Sandron

\section{Introduction}

1 Le département ultramarin de La Réunion est exposé à tous les risques naturels majeurs, sauf les avalanches (BRGM, 2016). Ces facteurs de vulnérabilité environnementale, qui peuvent peser sur le choix résidentiel voire sur la décision d'une mobilité résidentielle, se combinent à une série de facteurs de vulnérabilité propres aux personnes âgées largement décrits dans la littérature scientifique (Makdessi et al., 2017 ; Michel et al., 2017), auxquels s'ajoutent les conditions de vie des personnes âgées réunionnaises, particulièrement difficiles eu égard aux caractéristiques économiques, sociales et sanitaires de cette partie de la population, née pour la plupart à une époque où sévissait encore le paludisme sur l'île (Sandron, 2007 ; Beaugendre et al., 2014 ; Klein et Sandron, 2016).

Dans ce contexte, l'objet de cette contribution est de déceler l'aspect cumulatif des facteurs de vulnérabilité des personnes âgées potentiellement induits par une mobilité résidentielle récente, et en retour les facteurs de vulnérabilité à l'origine d'une mobilité résidentielle. Nous évoquerons ces facteurs de vulnérabilité à l'échelle individuelle, notamment dans les registres économique, sanitaire, social et environnemental.

3 L'argumentation sera fondée sur un état des lieux macroscopique des conditions de vie des personnes âgées à La Réunion et sur l'analyse d'un corpus de quatorze entretiens 
semi-directifs menés auprès de personnes âgées à La Réunion en 2015. Ces entretiens ont été réalisés dans le cadre du programme de recherche AMARE (Ancrage et Mobilité Résidentielle à la Retraite) (voir Encadré) initié en 2015 et qui consistait à réaliser une enquête qualitative permettant de relier les mobilités à la retraite aux parcours de vie des individus sur plusieurs terrains en France (Gallou et al., 2019a). Le critère d'inclusion dans le panel d'individus retraités interrogés était d'avoir connu un changement d'adresse au cours de l'année 2014.

Pour présenter ce travail, une première section contextualisera brièvement l'environnement de vie des habitants de La Réunion pour mieux en saisir les facteurs de vulnérabilité issue des spécificités géographiques, physiques, économiques, sociales et sanitaires. Une deuxième section se centrera ensuite sur les facteurs de vulnérabilité des personnes âgées réunionnaises dans l'optique de mieux comprendre à un niveau macroscopique leurs potentiels liens avec les mobilités résidentielles. Sur la base de notre corpus d'entretiens à La Réunion, une troisième section détaillera les facteurs de vulnérabilité des personnes âgées ayant entraîné une mobilité résidentielle ou induisant un désir de mobilité. En retour, l'impact de la mobilité résidentielle sur la vulnérabilité des personnes âgées sera évoqué. Enfin, la conclusion mettra en parallèle les effets synergiques de la vulnérabilité des personnes âgées interrogées avec leur mobilité résidentielle et plaidera pour une approche fine au niveau des territoires pour mieux en comprendre les ressorts.

\section{Encadré. AMARE (Ancrage et Mobilité Résidentielle à la Retraite)}

Il s'agit d'un projet de recherche coordonné par l'Unité de recherche sur le vieillissement de la CNAV (Caisse nationale d'assurance vieillesse) qui associe l'université de Tours (laboratoire CITERES), l'Ined (Institut national d'études démographiques) et l'IRD (Institut de recherche pour le développement). À partir des fichiers de la CNAV, une centaine de personnes retraitées ont été sélectionnées pour réaliser une première série d'entretiens. Plusieurs groupes de retraités ont été distingués en fonction de l'ancienneté de la retraite et de l'âge. Les situations de retraités ayant été récemment mobiles (au cours de l'année 2014 ou 2015) ont été analysées en tenant compte des parcours de vie, des positions sociales, du genre, des situations conjugales, du réseau familial, du parcours résidentiel, des projets de mobilité et du rapport au vieillissement. Le but du programme était de " collecter des données sur le parcours résidentiel des retraités, sur le logement actuel (description, motif de déménagement...), sur l'influence éventuelle des situations familiales et conjugales sur la mobilité, sur les rapports au nouvel environnement (activités quotidiennes, relations sociales et solidarités), sur les projets (ou non) de mobilité, et enfin sur la perception du vieillissement par l'enquêté » (Gallou et al., 2019a, p. 224). Le terrain a été mené sur trois territoires différents : l'île-de-France, l'Indre-et-Loire et La Réunion. D'un point de vue méthodologique, des personnes de plus de 60 ans ont été tirées au sort (sur le critère de mobilité, de sexe et d'âge) dans la base de données de la CNAV. Un questionnaire ayant pour objectif de vérifier que leur profil correspondait bien à la recherche et de demander leur accord pour faire un entretien (une prise de contact était prévue par téléphone) leur a été envoyé. Différentes vagues d'envoi ont été effectuées afin d'obtenir le nombre d'entretiens et les caractéristiques d'enquêtés souhaitées. Sur le terrain réunionnais, les retours des questionnaires 
nous ont permis de contacter une trentaine de personnes, et parmi elles seize entretiens ont pu être menés. Finalement, quatorze entretiens ont été retenus car les deux autres ne répondaient pas aux critères du corpus (les deux personnes interrogées n'avaient pas connu de mobilité résidentielle au cours de la dernière année). La principale limite méthodologique réside dans le mode de recrutement des enquêtés, certaines personnes ont pu penser que leur participation à l'enquête pourrait avoir un effet sur les aides sociales qu'elles perçoivent.

Tableau récapitulatif des caractéristiques des enquêté.es (noms modifiés)

\begin{tabular}{|c|c|c|c|c|c|c|c|c|c|c|}
\hline Pseudo & \begin{tabular}{|l} 
Date \\
entretien \\
(min)
\end{tabular} & CSP ego & $\begin{array}{l}\text { CSP } \\
\text { conjoint.e }\end{array}$ & Âge ego & $\begin{array}{l}\text { Âge } \\
\text { conjoint.e }\end{array}$ & Couple & $\begin{array}{l}\text { Nombre } \\
\text { d'enfants } \\
\text { en vie }\end{array}$ & \begin{tabular}{|l|} 
Bénéficie \\
d'une aide \\
(hors \\
Aspa)
\end{tabular} & $\begin{array}{l}\text { Commune } \\
\text { de } \\
\text { résidence }\end{array}$ & \begin{tabular}{|ll} 
Motif & principal \\
du & \\
déménagement
\end{tabular} \\
\hline Mme Bretel & 112 & Employée (cantine) & $\mathrm{ne}$ & 78 & & Non & 2 & Non & Saint-Pierre & $\begin{array}{l}\text { Rapprochement } \\
\text { du fils }\end{array}$ \\
\hline Mme Hoarau & 73 & \begin{tabular}{|l} 
Employée \\
(gardienne)
\end{tabular} & $\mathrm{nc}$ & 60 & & Non & 2 & Oui & Saint-Denis & $\begin{array}{l}\text { Appartement } \\
\text { personnel }\end{array}$ \\
\hline M. Damiano & 68 & $\begin{array}{l}\text { Employée } \\
\text { (bâtiment) }\end{array}$ & Employée & 75 & -35 & Oui & 4 & Oui & Saint-Paul & Plus d'espace \\
\hline M. Lalou & 30 & Cadre (bâtiment) & Sans activité & 82 & 82 & Oui & 4 & Oui & Petite-fle & $\begin{array}{l}\text { Don de la maison } \\
\text { familiale }\end{array}$ \\
\hline M. Prade & 62 & $\begin{array}{l}\text { Chef d'entreprise } \\
\text { (automobile) }\end{array}$ & $\mathrm{nc}$ & 68 & 65 & Oui & 1 & Non & Saint-Pierre & $\begin{array}{l}\text { Récupération } \\
\text { logement }\end{array}$ \\
\hline Mme Barton & 56 & Employée & Employée & 72 & 72 & Oui & 1 & Non & Saint-Denis & $\begin{array}{l}\text { Rapprochement } \\
\text { du fils }\end{array}$ \\
\hline Mme Divoux & 52 & Employée & $\mathrm{ne}$ & 65 & & Non & 11 & Oui & Saint-Pierre & Dispute fille \\
\hline M. Fontaine & 125 & $\begin{array}{l}\text { Employée } \\
\text { (bâtiment) }\end{array}$ & ne & 70 & $\mathrm{nc}$ & Oui & 5 & Oui & Le Tampon & $\begin{array}{l}\text { Appartement } \\
\text { personnel }\end{array}$ \\
\hline M. Gilbert & 49 & Employée (routier) & ne & 70 & nc & Oui & 5 & Oui & Cilaos & $\begin{array}{l}\text { Température } \\
\text { clémente }\end{array}$ \\
\hline M. Bara & 88 & $\begin{array}{l}\text { Cadre (labo } \\
\text { pharmaceutique) }\end{array}$ & ne & 68 & & Non & 0 & Non & Saint-Pierre & $\begin{array}{l}\text { Appartement } \\
\text { personnel }\end{array}$ \\
\hline M. Chong & 54 & $\begin{array}{l}\text { Commerçant, } \\
\text { artisan }\end{array}$ & Commerçant & 67 & & Non & 6 & Oui & Saint-Pierre & \begin{tabular}{|l} 
Retour domicile \\
après séparation
\end{tabular} \\
\hline M. Franck & 58 & Ouvrier & $\mathrm{nc}$ & 79 & 79 & Oui & 5 & & Le Port & \begin{tabular}{|l|}
$\begin{array}{l}\text { Vente ancien } \\
\text { appartement }\end{array}$ \\
\end{tabular} \\
\hline Mme Durel & 73 & Employée & $\mathrm{nc}$ & 65 & & Non & 1 & Non & Saint-Leu & Changer de vie \\
\hline Mme Grand & 41 & Employée & nc & 69 & & Non & 2 & & Saint-Paul & $\begin{array}{l}\text { Habiter chez sa } \\
\text { fille }\end{array}$ \\
\hline
\end{tabular}

\section{Les vulnérabilités du territoire réunionnais}

5 Située à 9000 kilomètres de la France métropolitaine, d'une superficie de $2500 \mathrm{~km}^{2}$, l'île de La Réunion est un Département français depuis 1946, une Région française depuis 1982 et une Région ultrapériphérique européenne (RUP) depuis 1997. Peuplée de 860000 habitants en 2019, elle affiche la troisième densité régionale de France. La Réunion est soumise à dix des onze risques majeurs naturels prévisibles recensés en France (inondations, séismes, éruptions volcaniques, mouvements de terrain, avalanches, cyclones, tempêtes, fortes houles, marées de tempête et tsunamis), ce qui en fait le département français le plus exposé (BRGM, 2016). Elle est aussi concernée par l'ensemble des cinq risques industriels majeurs prévisibles existant en France (nucléaire, industriel, transport de matières dangereuses, rupture de barrage, rupture de digue).

6 L'exiguïté du territoire, son insularité, son éloignement de la métropole et des autres territoires, la concentration de la population et de l'activité sur les zones littorales sont d'autres variables physiques et géographiques de vulnérabilité à La Réunion (Arnell 
et al., 2018). Celles-ci influent de manière négative sur la situation économique à cause de la nécessité d'importer une grande partie des produits de consommation et des ressources énergétiques avec un coût d'approvisionnement élevé (Bertrand et Richard, 2010). Malgré un certain dynamisme de la croissance, l'économie réunionnaise se révèle relativement fragile en raison d'une instabilité des prix, d'un taux de chômage structurel élevé et d'un déficit chronique de la balance commerciale (Goavec et Hoarau, 2015). En 2018, le PIB par habitant est de 21500 euros à La Réunion (34 300 euros en France) tandis que le taux de chômage au sens du BIT y est égal à $24 \%$ (9 \% en France) (IEDOM, 2019). Le chômage est l'une des explications de la forte proportion de Réunionnais vivant sous le seuil de pauvreté : 38 \% contre $14 \%$ en métropole (Besson, 2020). La proportion des bénéficiaires de la CMU-C (couverture maladie universelle complémentaire) est cinq fois plus élevée à La Réunion qu'en métropole. Ce sont $37 \%$ des Réunionnais qui en bénéficient (PIES, 2017).

7 Les conséquences de ces facteurs de vulnérabilité économique et sociale sont néanmoins atténuées par une politique de transfert de la part des pouvoirs publics, jugée relativement efficace au regard de la vulnérabilité structurelle de l'économie réunionnaise (Goavec et Hoarau, 2015). Ceci est d'autant plus important qu'ils sont à leur tour corrélés avec des facteurs de vulnérabilité sanitaire ou tout au moins des indicateurs de santé d'un niveau inférieur à ceux de la métropole. Ainsi, l'espérance de vie à la naissance des hommes réunionnais en 2018 est de 77,6 ans contre 79,6 ans en métropole, celle des femmes réunionnaises de 84,0 ans contre 85,5 ans en métropole (Sui-Seng, 2020). La mortalité infantile y est presque deux fois plus élevée qu'en métropole (6,5 décès pour 1000 enfants nés vivants vs 3,6 en métropole). Selon SuiSeng $(2020$, p. 2$)$, «cela pourrait s'expliquer par des conditions socio-économiques moins favorables sur l'île, davantage de facteurs à risque pesant sur les grossesses, et une entrée plus tardive des femmes enceintes dans le parcours de santé prénatale». L'état de santé perçu est légèrement moins bon à La Réunion qu'en métropole puisque $64 \%$ des Réunionnais déclarent en 2014 un bon état de santé général contre $69 \%$ des Français métropolitains (PIES 2017). D'autres indicateurs témoignant d'une certaine vulnérabilité sanitaire à La Réunion se lisent à travers des délais d'attente moyens supérieurs à un mois pour les consultations dans certaines spécialités médicales (cardiologie, diabétologie, dermatologie, orthopédie, ophtalmologie), la persistance de maladies infectieuses comme la dengue et le chikungunya, un accès aux soins difficiles pour les habitants des zones enclavées dans les hauteurs de l'île, le renoncement aux soins pour raisons financières pour un Réunionnais sur six (Besson et Caliez, 2016; PIES, 2016).

\section{Vulnérabilités dans la vieillesse et mobilité résidentielle à La Réunion : approche macroscopique}

8 Après avoir décrit les facteurs de vulnérabilité géophysique du territoire réunionnais et les facteurs de vulnérabilité socioéconomique de sa population, nous nous intéressons à présent aux facteurs de vulnérabilité des personnes âgées. Ceci nous permettra de mieux appréhender les incidences croisées de la mobilité résidentielle et des facteurs de vulnérabilité des personnes âgées sur le territoire réunionnais.

Dans une analyse des références bibliographiques concernant la vulnérabilité des personnes âgées, Makdessi et al. (2017) ont pu mettre en avant les principaux termes 
qui s'y trouvaient associés. Ainsi, un premier axe décline les aspects médicaux (pathologies cognitives, démence, délires, handicaps, comorbidités, anxiété, dépression, cancer) tandis qu'un second regroupe un ensemble de variables mobilisées dans les sciences sociales et ayant trait aux aspects physique, économique, sanitaire et social évoqués auparavant (résilience, risque, environnement, changements climatiques, santé publique, santé mentale, dimensions économiques). Cette recension rejoint l'analyse de Oris (2017) qui rappelle l'origine environnementaliste du concept de "vulnérabilité » en dissociant la probabilité de survenue d'un événement de ses conséquences. Selon cette perspective, après la confrontation à un risque, à un événement, un individu peut en sortir affaibli, stable ou renforcé.

La question est donc de savoir comment les ressources propres d'une personne vont lui permettre de réagir face à une situation de stress. Ceci va dépendre de ses « capacités à mobiliser des ressources individuelles et collectives " (Michel et al., 2017). C'est là qu'intervient alors la fragilité qui va affecter le stock et les capacités de mobilisation de ces ressources. La fragilité dépend à la fois de variables individuelles (âge, état de santé, présence ou non de déficiences physiques ou psychologiques) et de « facteurs exogènes, à savoir des éléments qui déterminent l'interaction du sujet âgé avec son environnement social» (Albérola et al., 2016, p. 100). Or, que ce soit au sens d'une fragilité davantage physiologique ou d'une fragilité étendue à la sphère sociale, l'avancée en âge est un critère défavorable. Comme le souligne Thomas $(2019$, p. 86) : " fragilité et vulnérabilité se conjuguent pour saper la résilience ».

11 Vulnérabilité et fragilité vont donc de pair. Pour la thématique qui nous intéresse, si une personne âgée effectue une mobilité résidentielle, surtout si elle est subie, mais pas seulement, celle-ci peut être un événement générateur de stress et donc influer négativement sur sa vulnérabilité. Les différentes formes de capital (humain, économique, social, etc.) et les rapports sociaux vont définir des possibilités de mobilisation des ressources disponibles différentes selon les personnes et agir sur son niveau de vulnérabilité. Ceci est d'autant plus vrai que la personne âgée en question présente déjà des facteurs de fragilité, ce qui nous amène à traiter brièvement des conditions de vie des personnes âgées à La Réunion.

12 Entre 2013 et 2050, la proportion de personnes âgées de 60 ans et plus dans la population totale à La Réunion devrait doubler, passant de $14 \%$ à $27 \%$. Leur nombre passerait de 115000 personnes en 2013 à 289000 personnes en 2050 selon le scénario central des projections de l'INSEE (Merceron, 2017). Durant la même période, la part des 75 ans et plus ferait plus que tripler, passant de $4 \%$ à $13 \%$ de la population totale.

13 Nous l'avons vu, l'espérance de vie est inférieure de presque deux ans à La Réunion par rapport à la métropole. La dépendance des personnes âgées est aussi plus précoce à La Réunion, avec cette particularité que même les personnes âgées dépendantes vivent très souvent à domicile ( $92 \%$ d'entre elles) y compris dans les cas de dépendance lourde puisque $83 \%$ des Réunionnais restent dans leur domicile, ce qui n'est le cas que de $49 \%$ des personnes âgées en métropole (Chaussy et Fabre, 2014).

En 2014, 59 \% des personnes âgées réunionnaises vivant à domicile déclarent une maladie chronique, cette prévalence augmentant avec l'âge (Caliez, 2018). Une personne âgée sur trois (32\%) déclare au moins un type de limitation fonctionnelle, c'est-à-dire des difficultés à effectuer certaines tâches usuelles de la vie quotidienne. Les limitations fonctionnelles physiques sont plus courantes $(26 \%)$ que les autres (sensorielles et cognitives). Elles affectent la capacité de marcher, monter les escaliers, 
lever le bras, se pencher, etc. Là encore, l'avancée en âge augmente considérablement l'occurrence des limitations fonctionnelles. Elles ont un retentissement fort sur les manières d'habiter, d'aménager son logement, voire d'entreprendre une mobilité résidentielle. Un autre facteur de santé notable en ce sens réside dans un résultat important de l'enquête Gramoune Care menée en 2016-2017 à La Réunion : le risque de dépression chez les Réunionnais de 65 ans et plus est de $46 \%$, chiffre relativement élevé (Caliez et al., 2018). Ces troubles de l'humeur et cet état de tristesse sont eux aussi des facteurs de fragilité ayant des retentissements sur les projets de mobilité résidentielle et sur la manière de réagir à la suite d'une mobilité résidentielle.

Quant au montant des revenus financiers des personnes âgées à La Réunion, il est largement inférieur à celui de leurs homologues en métropole. En 2013, le revenu annuel médian des personnes âgées de 65-74 ans à La Réunion est de 13502 euros contre 22734 euros en métropole. La différence est encore plus importante au sein de la tranche d'âge des 75 ans et plus, avec des revenus annuels médians respectifs de 10174 euros à La Réunion et de 19536 euros en métropole (Guéry, 2017). Un tiers des personnes âgées de 65 ans et plus et la moitié des personnes de 65 ans et plus vivant seules déclarent des ressources financières inférieures à 800 euros par mois au sein de leur foyer et un quart d'entre elles déclarent "y arriver difficilement" (Caliez et al., 2018). Avec une personne âgée sur deux vivant en 2013 sous le seuil de bas revenu (1 000 euros par mois), la précarité financière est clairement à La Réunion un facteur de vulnérabilité limitant l'éventail des possibilités de mobilité résidentielle.

Ajoutons qu'à La Réunion, la scolarisation ayant été massivement orientée vers le primaire jusqu'en 1970, les personnes aujourd'hui âgées de 75 ans et plus sont très peu diplômées, 90 \% d'entre elles n'ont aucun diplôme ou ont le niveau Brevet des collèges (Guéry, 2017). En 2011, près de $40 \%$ des Réunionnais âgés de 60-65 ans étaient en situation d'illettrisme.

17 Enfin, un facteur de fragilité supplémentaire provient de la topographie de l'île de La Réunion, très pentue et escarpée, où les déplacements ne peuvent se faire qu'en voiture ou en transport en commun. Or, seulement $32 \%$ des Réunionnais de plus de 60 ans possèdent un véhicule, les autres rencontrant donc des difficultés de mobilité au quotidien (Guéry, 2017). Ce facteur est évidemment important dans les choix résidentiels, qui plus est dans un territoire au climat tropical (forte chaleur, averses) qui ne favorise pas les déplacements à pied.

18 Avant d'analyser plus spécifiquement les relations entre ces différents facteurs de fragilité et la mobilité résidentielle à partir des entretiens semi-directifs, il importe de revenir sur les conditions de logement des personnes âgées à la Réunion.

De prime abord, la situation de logement des personnes âgées à La Réunion paraît plutôt bonne. En effet, elles sont en grande majorité propriétaires de leur logement, qui est dans neuf cas sur dix une maison individuelle (appelée case ou kaz en créole) de grande dimension. Ainsi, $65 \%$ d'entre elles sont des F 4 ou F5 et $22 \%$ des F3. Même si ces grands logements sont une source de charge accrue de travail domestique, l'enquête Gramoune Care a montré qu'en 2016-2017, huit personnes âgées sur dix considèrent que la taille de leur logement est satisfaisante (Caliez et al., 2018). Néanmoins, le confort n'y est pas toujours présent : $12 \%$ des personnes âgées n'ont pas accès à l'eau chaude, cette proportion atteignant $23 \%$ pour les hommes vivant seuls (Guéry, 2017). intéressés, leurs proches ainsi que les pouvoirs publics. De manière corrélative, l'offre 
d'hébergement collectif est largement inférieure à La Réunion (44 places pour 1000 personnes âgées de 75 ans et plus) comparativement à la métropole (125 places pour 1000 personnes âgées de 75 ans et plus) (Bouquet, 2018). Alors que $10 \%$ des personnes âgées de 75 ans et plus vivent en institution en métropole, seulement $4 \%$ des Réunionnais de la même tranche d'âge sont concernés (Guéry, 2017). Une spécificité dans les modes de résidence des personnes âgées à La Réunion est une offre importante d'hébergement de la part des familles d'accueil, $\mathrm{y}$ compris pour les personnes âgées très dépendantes (Klein et Sandron, 2018).

21 En matière d'entourage social, mentionnons que $34 \%$ des personnes âgées de 60 ans et plus vivent seules, $45 \%$ résident dans un ménage de deux personnes (dans $67 \%$ des cas avec leur conjoint et $20 \%$ avec l'un de leur enfant) tandis que $20 \%$ corésident avec au moins deux autres personnes. Les personnes vivant seules déclarent plus que les autres avoir de nombreuses difficultés pour leur autonomie, cela concerne $16 \%$ d'entre elles contre $10 \%$ et $11 \%$ respectivement pour les deux autres catégories (Caliez, 2018). Pour l'ensemble de la France, ce sont $6,3 \%$ des personnes de 60 ans et plus vivant à domicile qui sont en situation de perte d'autonomie.

Au total, selon l'enquête Gramoune Care, huit personnes âgées sur dix déclarent être satisfaites de leur mode de vie actuel et désirent rester dans leur domicile, avec cependant des aménagements souhaités pour la moitié d'entre elles. Seules $2 \%$ des personnes âgées envisagent de quitter leur domicile pour un hébergement en établissement ou en famille d'accueil (Caliez et al., 2018).

\section{Vulnérabilités dans la vieillesse et mobilité résidentielle à La Réunion : approche individuelle}

Les facteurs de vulnérabilité des personnes âgées réunionnaises tels que nous venons de les présenter donnent une vision générale des potentialités mais aussi des éventuels freins à la mobilité résidentielle. Cette population, plutôt défavorisée par rapport aux tranches d'âge actif à La Réunion et par rapport aux personnes âgées en France métropolitaine, aspire très majoritairement à poursuivre sa vieillesse à domicile, hors des hébergements en institution. Les facteurs de vulnérabilité évoqués peuvent jouer dans deux directions quant au souhait et à la concrétisation d'une mobilité résidentielle. D'un côté, ils peuvent jouer en faveur d'une mobilité résidentielle du fait de l'accumulation des problèmes et des limitations dans l'habitat et l'environnement courants, de l'autre, ils peuvent empêcher la réalisation d'un projet de mobilité résidentielle perçu comme trop coûteux, trop compliqué ou trop contraignant.

Pour mieux saisir les interactions entre mobilité résidentielle et vulnérabilités des personnes âgées, nous proposons de nous appuyer sur le corpus constitué de quatorze entretiens semi-directifs réalisés à la Réunion au cours de l'année 2015 dans le cadre du programme de recherche AMARE.

Ceci nous permettra de traiter des facteurs et des situations de vulnérabilité à l'origine des mobilités résidentielles ainsi que des retentissements de ces mobilités sur la vulnérabilité des personnes âgées. L'impact de la mobilité résidentielle sur la vulnérabilité des personnes âgées interrogées durant nos entretiens sera mesuré à l'aune de leur volonté de changer à nouveau de domicile, alors qu'ils ont emménagé très récemment. Dans cette double perspective, nous retiendrons donc davantage les 
variables mettant en exergue des situations individuelles de fragilité et de vulnérabilité, c'est-à-dire que nous privilégierons un axe de recherche qui souhaite rendre compte $\mathrm{du}$ fait que les comportements résidentiels sont plus ou moins contraints et oppose ceux qui sont choisis à ceux qui sont subis (entendons par là des comportements contraints par la faiblesse des ressources financières, par un mauvais état de santé ou encore imposés par un tiers, par exemple à l'occasion d'une opération de renouvellement urbain ou lorsqu'un enfant incite son parent âgé à entrer en maison de retraite). (Caradec, 2010, p. 2)

Il semble établi que c'est l'accumulation des facteurs de vulnérabilité qui déclenche l'état de vulnérabilité à proprement parler, au même titre que la polypathologie favorise la sarcopénie (diminution des capacités musculaires) chez les personnes âgées. Pour rendre compte à la fois des effets synergiques des facteurs de vulnérabilité entre eux et de la diversité de ces facteurs, nous avons opté pour présenter, de manière duale, une situation personnelle complète ${ }^{1}$ et des extraits de verbatim par grands thèmes. Les facteurs sous-jacents à la vulnérabilité, que ce soit en amont ou en aval d'une mobilité résidentielle, reprennent dans leur large majorité ceux évoqués précédemment dans les sphères de l'environnement et du territoire, de l'économie, du social, de la santé, de l'entourage familial et relationnel.

Enfin, puisqu'il s'agit de traiter des facteurs de vulnérabilité, les mobilités résidentielles des personnes âgées dont les motivations sont davantage hédonistes, correspondant à une volonté d'améliorer leur bien-être en choisissant l'héliotropisme et/ou des lieux de vie avec davantage d'aménités n'ont pas été pas abordées ici, même si ces situations existent aussi.

\section{1) Facteurs de vulnérabilité relevés dans les entretiens semi- directifs}

Puisque nous nous intéressons aux facteurs de vulnérabilité, il s'agit ici de traiter davantage des mobilités d'ajustement plutôt que des mobilités de confort (Caradec, 2010 ; Nowik et Thalineau, 2010). Selon Caradec (2010, p. 11) :

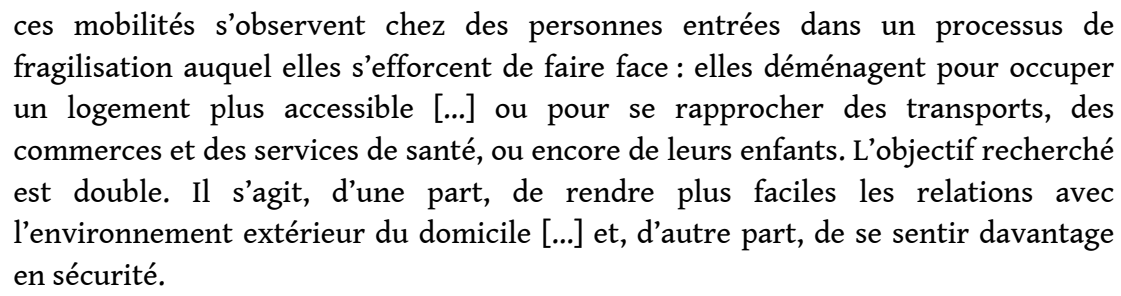

29 Cet aspect sécuritaire touche aussi bien les domaines de la santé et des faiblesses physiologiques et musculaires que celui de la sécurité directe (agression, vol, etc.). Il est souvent renforcé par un autre facteur déterminant dans les choix de résidence qui est celui du sentiment de solitude. Nous retrouverons dans les verbatim des personnes interrogées les deux composantes que sont la solitude liée à l'absence de relations sociales et celle consécutive à un isolement géographique (Nowik, 2018).

Précisons que la recherche d'un nouveau lieu de vie est néanmoins «à double tranchant " (Caradec, 2010), dans la mesure où le départ du lieu précédent constitue une perte de repères et que ce lieu comprenait sans doute aussi des éléments positifs qui ne seront pas présents dans le nouveau lieu de vie. C'est pourquoi les facteurs de vulnérabilité se retrouvent aussi bien en amont qu'en aval de la mobilité résidentielle. 
31 Primo, dans nos entretiens semi-directifs, les facteurs de vulnérabilité recensés peuvent être de nature économique, liés au niveau de vie. Un déménagement est fréquemment causé par l'inadéquation entre les revenus perçus et les dépenses occasionnées par la situation de résidence (loyer, déplacements). Inversement, le manque de revenu est souvent évoqué comme un frein majeur à tout nouveau projet de mobilité résidentielle, alors que celui-ci serait souhaité.

32 C'est précisément la situation que rencontre Monsieur Fontaine, 70 ans, divorcé et ancien employé du bâtiment :

Je paye 480 euros mon logement, 480 euros, j'ai 82 euros d'aide au logement, je trouve que c'est un peu faible, qu'est-ce que je peux faire? Alors si je vous dis par exemple sur ma retraite quand je tire l'assurance de voiture 80 euros et 50 centimes, assurance-décès 40 euros et 50 centimes, assurance santé 10 euros, merci à Dieu je gagne les chèque santé, je paye Internet téléphone 55 euros et 90 centimes [...] Je suis obligé de demander aux enfants de m'aider. Il y en a une qui m'aide, mais sans quoi, qu'est-ce qui reste à manger? Il n'y a plus rien, on vit comme ça. Depuis le $1^{\text {er }}$ mars 2014, j'étais chez ma fille, avant j'ai fait une demande de logement social pour baisser les tarifs.

L'exemple de M. Bara, 68 ans et célibataire, montre que la vulnérabilité économique pousse également certaines personnes à immigrer à Madagascar, pays voisin où le prix de la vie est très inférieur à celui de la France. Le profil des interviewés qui envisagent, ou mettent en œuvre ces migrations, sont généralement des hommes qui y ont déjà vécu dans le passé et qui ont des ressources économiques très faibles, ne permettant pas, selon leur perception, de « vivre décemment en France »:

J'ai des revenus très faibles et je me rends compte que je dépense le double de ce que je reçois donc ça ne peut pas durer. Donc j'ai pris la décision d'aller m'installer à Madagascar et j'y vais au mois d'août. Je n'y vais pas comme ça, à l'aveugle, j'ai des amis qui sont là-bas, j'étais il y a un mois à Madagascar vingt-cinq jours, on n'avait pas besoin de visa, j'ai fait les démarches, je suis allé au Consulat, je sais qu'ils acceptent les retraités français, donc je voudrais m'installer à Diego et je pense pouvoir vivre avec de petits revenus. J'ai à peine 1000 euros par mois, je ne peux pas vivre ici avec ça, j'ai une voiture, je paye 500 euros pour cet appartement, ça ne peut pas durer comme ça.

34 Cette décision, logique sur le plan économique, peut néanmoins être risquée pour des personnes âgées dans la mesure où le système de santé à Madagascar est bien moins performant qu'en France.

Certains projets de mobilité sont en revanche freinés par les coûts engendrés. C'est le cas de Madame Hoarau, 60 ans, célibataire, qui souffre de l'éloignement de ses proches, restés en métropole, et de l'absence de relations et d'interactions sociales à La Réunion :

Je pense retourner en métropole parce que mes amis sont là-bas, mes sœurs et mes frères sont là-bas, je suis seule ici à La Réunion. J'y pense mais je n'ai pas les moyens, il faudrait que je parte avec vingt-trois kilos de bagages, ça il faut y penser, on n'y pense pas. Financièrement, matériellement, je ne peux pas partir, ici c'est vraiment carcéral.

Secundo, les facteurs de vulnérabilité liés à l'environnement domestique ou territorial sont très fréquents dans les verbatim des personnes âgées. Ils viennent s'ajouter aux autres facteurs de vulnérabilité et notamment à ceux liés à la santé. Ainsi, une personne souffrant des jambes ou dépressive aura d'autant moins d'entrain à affronter 
les difficultés de mobilité quotidienne comme un chemin en pente pour aller faire ses courses.

Je retourne dans l'Est, je vois toute cette verdure, c'est verdoyant, c'est beau. Il pleut, $\mathrm{j}$ 'aime bien cette pluie, température et tout, $\mathrm{j}$ 'aimais beaucoup vivre là-bas, mais je ne pouvais pas, Bras-Panon ce n'est pas possible, il fallait une heure à pied pour aller à la poste, vingt minutes de marche pour le bus au Car Jaune, et encore maintenant les arrêts ont été supprimés, donc trois quarts d'heure de marche ce n'est pas possible. (Madame Hoarau, 60 ans, divorcée)

Les caractères physiques et géographiques de l'île de La Réunion ressortent souvent lorsqu'il s'agit du sentiment d'isolement des personnes âgées résidant hors des centres urbains. À l'origine, ne pas disposer de voiture est fréquemment mentionné, notamment par Madame Durel :

Je ne dis pas que je suis en train de crever de faim, parce que mon logement n'est pas cher, je paye 150 euros électricité comprise, mais c'est le coin, ça c'est trop, trop retiré. Quelqu'un qui ne conduit pas, c'est catastrophique. S'il avait fallu, je serais allée à la mairie pour m'inscrire pour un autre logement, là je m'en vais donc ça va, mais sinon je ne peux pas

ou encore de Monsieur Chong :

En arrivant ici, j'ai pris une voiture, c'est pour ça, je paye 221 euros tous les mois, c'est pour ça le crédit, l'argent de la retraite, il va dans les crédits, parce que je paye une voiture 221 euros par mois. Sans voiture, on ne peut pas ici, ma famille habite à Saint-Pierre, sans voiture je ne peux pas.

Cette quasi-obligation de disposer d'une voiture dans de telles conditions peut grever le budget et cet argument renvoie aux vulnérabilités économiques.

Inversement, l'espace urbain n'est pas toujours adapté aux personnes vieillissantes et celles-ci sont parfois obligées de déménager pour se retrouver dans des espaces plus adaptés. Monsieur Bara, ancien cadre dans un laboratoire pharmaceutique âgé de 68 ans et célibataire, nous dit:

Je suis arrivé à Saint-Denis et je me suis dit que je ne pouvais pas rester ici, ça me déplaisait par rapport à quand j'étais en activité, je ne reconnaissais plus rien [...] je n'arrivais plus à circuler, il y avait du monde partout, j'ai même eu une contravention. J'ai dit je ne reste pas ici, ce n'est pas possible. J'étais dans un petit hôtel à Saint-Denis et je suis resté là deux mois et demi je crois. J'ai sympathisé avec le patron et au bout d'un moment, j'ai dit que j'avais l'intention de rester à La Réunion, j'ai dit ça ne me plaît pas Saint-Denis et il me dit, eh bé Saint-Pierre.

41 Les facteurs précédents peuvent devenir rédhibitoires et aboutir à un changement résidentiel ou bien survenir à la suite d'un changement résidentiel dans la mesure où il faut souvent faire des concessions sur certains points dans le choix d'un nouveau logement. Ceci est particulièrement vrai à La Réunion, où la température est élevée sur le littoral et où le relief pentu et les routes escarpées peuvent être des obstacles pour les déplacements à pied, en voiture ou en transport en commun. Relief escarpé, fortes chaleurs ou fortes pluies, bâti peu dense dans les hauteurs, tous ces facteurs rendent quasi nécessaire soit la possession d'une voiture, ce qui entraîne des coûts, soit la possibilité de bénéficier de la voiture d'un proche, ce qui entraîne un sentiment de dépendance.

Tertio, les facteurs de vulnérabilité des personnes âgées se retrouvent couramment dans les registres de la santé et de la détresse psychologique, les deux étant fréquemment associés. Ainsi, la lassitude de Mme Bretel, 78 ans et veuve, est 
multimodale : elle se sent isolée, fatiguée et fragile, révélant un cumul des facteurs de vulnérabilité qu'il est difficile d'isoler. Elle annonce alors :

Je finirai ma vie à La Réunion, y'a pas à faire, je ne partirai plus, c'est trop de boulot. Je ne suis plus à un âge, même prendre l'avion, quand je suis avec mon fils, ça va je suis contente, je suis avec quelqu'un, mais prendre l'avion toute seule maintenant j'ai peur.

Outre le sentiment d'incapacité physique à vivre seule un nouveau déménagement, la décision de Madame Bretel de rester à La Réunion résonne comme une renonciation à améliorer sa situation personnelle.

Les propos de Madame Hoarau, ancienne gardienne d'immeuble qui a toujours vécu dans un contexte de grande précarité économique, témoignent d'une extrême fragilité et d'un sentiment de forte contrainte.

Je me sens seule, je me sens isolée, le sentiment d'avoir été abandonnée. Isolement parce que je ne peux pas bouger, je n'ai aucune mobilité, je ne peux même pas faire quelques kilomètres. J'ai le permis de conduire, je conduis la voiture de ma fille quand elle est fatiguée, mais non ce n'est pas la vie qu'il faut à quelqu'un qui est dans ma situation financière et familiale, moi je peux mourir là personne ne le saurait.

Quarto, les personnes âgées interrogées accordent une importance primordiale aux relations familiales et aux relations sociales. Ce sont des motivations fortes pour déménager, soit à cause de tensions soit au contraire pour se rapprocher d'un enfant ou d'un membre de la famille.

[Je suis revenu à La Réunion] pour retrouver mon origine et les amis, les amis d'enfance et tout, et j'aime bien ici La Réunion. Quand j'ai quitté l'hôpital, l'assistante sociale et le docteur m'ont trouvé un appartement à Saint-Pierre à Terre-Sainte mais il faisait une chaleur horrible, je ne pouvais pas résister, alors j'ai trouvé un copain et avec le bouche-à-oreille il m'a fait connaître cette dame et j'ai atterri là.

C'est donc la volonté de se rapprocher de ses amis d'enfance qui a poussé Monsieur Gilbert, 70 ans et divorcé, à revenir s'installer à La Réunion. Les conditions climatiques l'ont orienté à Cilaos, dans les hauteurs de l'île, où le climat est plus frais que sur la côte.

Pour Monsieur Lalou qui a 82 ans et qui est marié, c'est la cohabitation difficile avec son fils qui a poussé sa femme et lui à déménager même si cela les contraint davantage d'un point de vue économique.

On a habité à peu près à trois kilomètres d'ici, on habitait là-bas et on a laissé notre maison à notre fils qui habitait avec nous. On ne s'entendait pas très bien, on a préféré venir habiter ici, on a quitté notre maison, on est venus ici mais le loyer est cher, on est locataire, on n'a aucune aide.

Dans certains cas, la perte de contact avec les proches rend l'expérience de la mobilité douloureuse, comme pour Madame Divoux, 65 ans, séparée, qui vit très isolée et regrette aujourd'hui son choix.

Si j'avais su, je ne serais pas venue. Ma fille mon garçon, tout était avec moi, là tout d'un coup plus rien, là je me retrouve toute seule. Il y a des jours c'est dur, je pense à faire n'importe quoi, je pense des mauvaises choses, mais bon, je passe mon temps devant la télé vingt-quatre heures sur vingt-quatre, c'est très très très dur toute seule, c'est un cauchemar.

Que ce soit dans le domaine de la santé, du lien social, des revenus ou de l'environnement au sens large, les personnes âgées tentent ainsi de garder des « prises 
sur le monde extérieur " (Caradec, 2010). Cependant, dans le cas où trop de facteurs de vulnérabilité s'accumulent sur le lieu de vie, la mobilité résidentielle d'ajustement peut devenir la solution ultime. Le témoignage de Madame Bretel en est une illustration.

\section{2) Mobilité résidentielle et cumul des vulnérabilités : l'exemple de Madame Bretel}

Madame Bretel a 78 ans, elle est veuve depuis presque trente ans, c'est une ancienne employée qui a deux enfants. Elle est à la retraite depuis 2001. Fâchée avec son plus jeune fils, elle a déménagé à La Réunion en 2014 parce que son fils aîné y habite.

J'ai deux fils mais l'autre il ne me parle plus depuis quatorze ans, plus de contacts avec moi ni les petits-enfants. Il a trois enfants, j'ai tout essayé, j'allais voir un médiateur, j'allais voir des gens comme ça [...] mon deuxième fils m'a abandonnée [...] j'ai trop été malheureuse de mon fils qui m'a quittée, on ne peut pas s'imaginer un fils qui ne parle plus à sa mère.

Il ressort de l'entretien que c'est avant tout cette solitude et le manque de contact avec sa famille proche qui l'ont poussée à venir s'installer à La Réunion en 2014. Auparavant, Madame Bretel avait vécu vingt-cinq ans en région parisienne avec son mari, agent SNCF, décédé il y a une trentaine d'années. Peu après le début de sa retraite en 2001, elle a vécu ensuite cinq ans à Amiens, là où habitait sa sœur. Sans contact avec son fils cadet en métropole, éloignée physiquement de son fils aîné qui résidait à La Réunion, ayant connu «des ennuis » avec sa sœur, elle a emménagé dans le Sud de la France en 2008 pour bénéficier d'un climat ensoleillé et rejoindre son frère, dans l'espoir qu'il s'occupe d'elle. Cependant, celui-ci n'était pas vraiment présent ni "dévoué » à ses yeux, elle s'est alors sentie très seule. La prise de décision effective de déménager à La Réunion en 2014 a mûri durant six ans, Madame Bretel en avait parlé à son frère dès le début de son projet.

Une fois arrivée à La Réunion en 2014, sa préoccupation majeure est donc d'être à proximité de son fils aîné. De ce point de vue, Madame Bretel a réussi son projet de mobilité. Mais cette installation a généré aussi de nombreux problèmes qui ont impacté sa qualité de vie. Plus précisément, c'est le désir profond de Madame Bretel d'être près de son fils qui l'a poussée à faire des choix de logement et d'environnement peu adaptés à son état de santé. En effet, si son appartement est correct, il comprend un escalier alors que Madame Bretel a souvent mal aux jambes et rencontre des difficultés pour monter les marches. Il en est de même pour aller faire ses courses, son appartement est dans une zone pentue, ce qui la limite pour y aller à pied. $\mathrm{Ne}$ possédant pas de véhicule, elle est alors tributaire de son fils et de sa belle-fille, réduisant par là même sa mobilité et la plupart de ses activités.

Il y a le supermarché pas très loin, j'y vais des fois avec le caddie mais il y a une côte

à monter, une côte terrible, quand vous montez on est essoufflé et quand vous descendez vous êtes obligé de freiner. [...] Non, je n'ai pas encore pris le bus parce que là j'ai eu beaucoup mal aux jambes ces derniers temps, je n'arrivais plus à monter, j'ai peur de ne pas réussir à monter dans le bus.

Madame Bretel est aussi confrontée à une forme de vulnérabilité sociale dans la mesure où elle n'a pas réussi à tisser des liens d'amitié ou de voisinage sur place. Elle a l'impression, selon ses termes, de n'avoir rencontré personne « d'intéressant » depuis son arrivée. De plus, les visites à La Réunion des amis ou des membres de la famille de métropole qu'elle escomptait au moment de son départ ne semblent plus d'actualité. 
Ça fait sept mois que j'habite ici, il n'y a pas très longtemps je déprimais. Vous savez j'ai eu une sacrée déprime. [...] Les dix mille kilomètres de séparation, c'est assez dur, parce que là mon petit-fils devait venir [...] mais il ne vient pas parce qu'il va faire une formation. [...] En métropole, j'avais des amis quand même, ils m'ont dit on ira te voir et puis personne ne vient, ils ne vont pas faire dix mille kilomètres pour venir me voir alors je me suis sentie tellement loin que j'ai craqué. résidentielle à La Réunion. Elle se dit loin de tout, abandonnée, ne fait plus vraiment de projets. Elle est néanmoins tiraillée entre rester dans son appartement, déménager à La Réunion pour un appartement sans escalier, retourner en métropole pour se rapprocher de sa sœur et de son petit-fils, à savoir le fils de son fils aîné qui a fait sa scolarité à La Réunion et qui est maintenant en formation en métropole. Finalement, après sept mois de mobilité résidentielle à La Réunion, Madame Bretel se plaint toujours de solitude même si les relations avec son fils aîné qui habite à proximité sont bonnes. Alors que ce témoignage met en évidence la mobilité résidentielle comme une réponse à la recherche du bien-être à travers la proximité des proches, on peut voir que Madame Bretel souffre d'une impossibilité à stabiliser ses relations affectives et paraît ne jamais se sentir bien là où elle est.

\section{Conclusion}

L'existence de facteurs de vulnérabilité, aussi bien au niveau des territoires qu'à celui des personnes, influence les désirs et projets de mobilité résidentielle ainsi que leurs représentations. L'approche macroscopique des vulnérabilités des personnes âgées à La Réunion et l'analyse individuelle qualitative menée à partir d'entretiens semi-directifs aboutissent à des résultats complémentaires et concordants. Plusieurs enseignements peuvent être tirés de cette recherche.

Tout d'abord, à l'instar de nombreuses autres recherches, l'analyse des entretiens individuels présentée ici révèle qu'une mobilité résidentielle est bien plus qu'un simple déplacement, elle s'inscrit dans une dynamique sociale individuelle et collective (Gallou et al., 2019b).

Ensuite, si les enfants des personnes âgées demeurent un soutien essentiel dans la vieillesse, et surtout dans les départements d'outre-mer (Beaugendre et al., 2014), les reconfigurations démographiques (enfants eux-mêmes âgés, enfants moins nombreux), familiales (monoparentalité, divorce) et spatiales (migration) rendent néanmoins ces solidarités moins automatiques et certaines qu'auparavant. De surcroît, leur qualité et leur intensité évoluent à La Réunion comme l'indique à la fois la littérature scientifique issue d'analyse d'enquêtes et la teneur des verbatim des entretiens AMARE présentés ici.

Un troisième résultat général tiré des verbatim des personnes interrogées est que la mobilité résidentielle d'ajustement ne semble survenir que lorsque plusieurs facteurs de vulnérabilité viennent s'accumuler et se renforcer, c'est-à-dire quand les possibilités d'ajustement catégoriels (aménagement du logement, portage de repas à domicile, mobilisation épisodique des personnes aidantes, etc.) ne sont pas ou plus suffisantes, ainsi que l'a montré Caradec (2010). Au-delà de la nécessité de prendre en considération ces contraintes matérielles amenant à la mobilité, il serait opportun d'aller encore plus loin en s'intéressant aux façons de vivre les relations aux autres (famille, voisinage) et aux subjectivités sous-jacentes qu'il s'agirait d'objectiver sociologiquement.

Populations vulnérables, 7| 2021 
59 , à une époque de la vie où les questions de santé, la qualité du cadre de vie et la possibilité de maintenir des liens sociaux ont pris le pas sur les préoccupations professionnelles, les spécificités environnementales et territoriales, déclinées notamment dans la section 1 et illustrées au cours de l'analyse des verbatim, sont un élément clé dans la compréhension fine des processus menant ou non à la vulnérabilité des personnes âgées (Nowik et Thalineau, 2010). Ceci plaide pour la poursuite et l'approfondissement de la réflexion sur l'adaptation locale de la société au vieillissement de la population.

\section{BIBLIOGRAPHIE}

Albérola É. et al. (2016), « Bien vieillir, retraite, dépendance, fragilité des séniors.

Représentations, réalités et attentes de la population française vis-à-vis des institutions ", Paris, Credoc, Collection des Rapports du Credoc, $n^{\circ} 334,232 \mathrm{p}$.

Arnell G., Darnaud M. et Jasmin V. (2018), « Rapport informatif fait au Sénat au nom de la délégation sénatoriale aux outre-mer sur les risques naturels majeurs dans les outre-mer (volet relatif à la prévention, l'alerte et la gestion de l'urgence) », tome 1, $\mathrm{n}^{\circ} 688$, Paris, Sénat, $224 \mathrm{p}$.

Beaugendre C., Breton D. et Marie C.-V. (2014), « Trajectoires familiales et conditions de vie des 60 ans et plus dans les départements d'outre-mer ", XVIII ${ }^{\mathrm{e}}$ Colloque international de l'AIDELF Trajectoires et âges de la vie, Bari, Italie, 26-29 mai, $24 \mathrm{p}$.

Bertrand F. et Richard E. (2010), « Adaptation des territoires insulaires. Éléments de réflexion à partir de deux îles françaises (Ré et La Réunion) », Vertigo, vol. 10, n³, p. 1-18.

Besson L. (2020), « Niveaux de vie et pauvreté à La Réunion en 2017 », INSEE Flash La Réunion, $n^{\circ} 169$.

Besson L. et Caliez F. (2016), « Accès aux soins à La Réunion. 84000 Réunionnais à plus de 30 minutes des urgences », INSEE Analyses La Réunion, nº 19.

Bouquet M. (2018), « Les établissements d'hébergement pour personnes âgées à La Réunion en 2015 ", Infos Statistiques, n²8, Agence Régionale de Santé Océan Indien, 15 p.

BRGM (2016), « Dossier départemental des risques majeurs de La Réunion », Saint-Denis de La Réunion, Préfet de La Réunion, 120 p.

Caliez F. (2018), « Incapacités et pertes d'autonomie des personnes âgées à La Réunion », Infos Statistiques, $\mathrm{n}^{\circ} 28$, Agence Régionale de Santé Océan Indien, 14 p.

Caliez F. et al. (2018), «État de santé et conditions de vie des personnes âgées de 65 ans ou plus à La Réunion », In Extenso, n 6, Plateforme d'information des études en santé, ARS OI, ORS OI, Cire OI, $24 \mathrm{p}$.

Caradec V. (2010), « Les comportements résidentiels des retraités. Quelques enseignements du programme de recherche "Vieillissement de la population et habitat" ", Espace, Populations, Sociétés, $\mathrm{n}^{\circ} 1$, p. 29-40.

Populations vulnérables, 7 | 2021 
Chaussy C. et Fabre É. (2014), « Deux fois plus de personnes âgées dépendantes en 2030 à La Réunion ", INSEE Partenaires Réunion, $\mathrm{n}^{\circ} 29,6 \mathrm{p}$.

Gallou R. et al. (dir.) (2019a), Vers de nouvelles dynamiques de mobilité résidentielle à la retraite? L'enquête AMARE (Ancrage et Mobilité résidentielle À la REtraite), Les Cahiers de la CNAV, $\mathrm{n}^{\circ} 13,256 \mathrm{p}$.

Gallou R. et al. (2019b), « Appréhender les mobilités résidentielles à la retraite et leurs évolutions ", in Gallou R. et al. (dir.) (2019), Vers de nouvelles dynamiques de mobilité résidentielle à la retraite? L'enquête AMARE (Ancrage et Mobilité résidentielle À la REtraite), Les Cahiers de la CNAV Les Cahiers de la CNAV, $n^{\circ} 13$, p. 10-20.

Goavec C. et Hoarau J.-F. (2015), « Une mesure de la vulnérabilité économique structurelle pour une économie ultrapériphérique européenne : le cas de La Réunion ", Géographie, économie, société, vol. $17, \mathrm{n}^{\circ} 2$, p. $177-200$.

Guéry R. (2017), «Les personnes âgées à La Réunion », Tableau de bord, Saint-Denis de La Réunion, Observatoire régional de la santé Océan indien, $44 \mathrm{p}$.

IEDOM (2019), « La Réunion 2018 », rapport annuel, Saint-Denis de La Réunion, Institut d'émission des départements d'outre-mer, $216 \mathrm{p}$.

Klein A. et Sandron F. (2016), « La Réunion : un territoire spécifique en matière de prise en charge des personnes âgées dépendantes ", in En quête de territoire(s), Actes du $3^{\mathrm{e}}$ colloque international du CIST, 17-18 mars, Grenoble, p. 258-262.

Klein A. et Sandron F. (2018), « Une nouvelle famille ? L'accueil familial des personnes âgées à La Réunion », in Sanderson J.-P. et Sousa Gomes M.-C. (dir.), Famille et vieillissement : enjeux et défis des solidarités intergénérationnelles au Nord et au Sud, Paris, AIDELF, p. 5-24.

Makdessi Y., Vichnevskaia T. et Weber A. (2017), « Émergences et usages du concept de vulnérabilité des personnes âgées ", Populations vulnérables, n 3, p. 13-25.

Merceron S. (2017), « La population réunionnaise à l'horizon 2050. Autant de seniors que de jeunes », INSEE Analyses La Réunion, n² 29.

Michel H. et al. (2017), « Fragilité, vulnérabilité et ressorts de santé des personnes âgées. Points communs, différences et perspectives opérationnelles ", Populations vulnérables, n 3 .

Nowik L. (2018), «La déprise à l'aune des habitats intermédiaires pour personnes âgées », Gérontologie et société, vol. 40, n 155, p. 87-104.

Nowik L. et Thalineau A. (2010), « La mobilité résidentielle au milieu de la retraite : un cadre spatial structurant lié à des configurations sociales ", Espace, Populations, Sociétés, n 1, p. 41-51.

Oris M. (2017), « Les contours du concept de vulnérabilité », in Oris M et al. (dir.), Les populations vulnérables, Actes du XVI ${ }^{\mathrm{e}}$ colloque national de démographie de la CUDEP, Bordeaux, p. 12-18.

PIES (2016), « Recours aux soins à La Réunion », In Extenso, n 5, Saint-Denis de La Réunion, Plateforme d'information des études en santé, ARS OI, ORS OI, Cire OI, $13 \mathrm{p}$.

PIES (2017), « Situation sanitaire Réunion et Mayotte », Saint-Denis de La Réunion, Plateforme d'information des études en santé, Agence régionale de santé Océan indien, $169 \mathrm{p}$.

Sandron F. (2007), « Dynamique de la population réunionnaise », in Sandron F. (éd.), La population réunionnaise. Analyse démographique, Paris, Éditions de l'IRD, p. 27-41.

Sui-Seng S. (2020), « Bilan démographique 2018 à La Réunion », INSEE Flash La Réunion, n 170.

Thomas P. (2019), « Les personnes âgées et leur vulnérabilité réelle ou supposée », Trajectoires Humaines Transcontinentales, $\mathrm{n}^{\circ}$ 5, p. 82-94. 


\section{NOTES}

1. Certains détails de lieux de résidence ont été gommés pour préserver l'anonymat.

\section{RÉSUMÉS}

Sur la base d'une revue de la littérature et d'un corpus de quatorze entretiens semi-directifs, l'objet de cet article est de mettre en relation les facteurs de vulnérabilité du territoire réunionnais, ceux des personnes âgées de ce département français d'outre-mer ainsi que ceux éventuellement issus d'une mobilité résidentielle. Pour cela, une analyse macroscopique et une analyse individuelle sont proposées autour des facteurs de vulnérabilité dans les registres économiques, sanitaires, sociaux et environnementaux. Ces deux niveaux d'analyse indiquent la nécessité d'une approche territoriale fine pour mieux comprendre la vulnérabilité des personnes âgées et ses liens avec la mobilité résidentielle.

On the basis of a literature review and a corpus of fourteen semi-directive interviews, the aim of this article is to relate the vulnerability factors of the Reunion Island, those of the elderly in this French overseas department and those possibly caused by residential mobility. For this purpose, a macroscopic analysis and an individual analysis are proposed with vulnerability factors in the economic, health, social and environmental domains. These two levels of analysis indicate call for a fine territorial approach to better understand the vulnerability of old people and its interactions with a residential mobility.

\section{INDEX}

Keywords : Reunion Island, vulnerability, frailty, residential mobility, old people

Mots-clés : La Réunion, vulnérabilité, fragilité, mobilité résidentielle, personnes âgées

\section{AUTEURS}

\section{ARMELLE KLEIN}

Post-doctorante

Chercheuse associée au Ceped, Université de Paris, IRD

armelle.klein@ird.fr

\section{FRÉDÉRIC SANDRON}

Directeur de recherche

UMR Ceped, université de Paris, IRD

frederic.sandron@ird.fr 